\title{
Lipid and Apolipoprotein Compositions of Two Species of ApoA-I Containing Lipoproteins in Young Girls with Insulin-Dependent Diabetes Mellitus
}

\author{
TAKAO OHTA, RIE NAKAMURA, SOROKU NISHIYAMA, MIHOKO KODAMA, AND \\ ICHIRO MATSUDA \\ Departments of Pediatrics /T.O., R.N., S.N., I.M.J and Child Development [M.K.J, Kumamoto University \\ Medical School, Kumamoto City, Kumamoto 860, Japan
}

\begin{abstract}
Two species of lipoproteins containing apoAI (A-ILp), lipoprotein containing apoA-I and apoA-II (LpA-I/A-II), and lipoprotein containing apoA-I but no apoA-II were isolated from 12 girls with insulin-dependent diabetes mellitus (IDDM) and from 19 healthy controls using aftjnity chromatography. When characterizing the lipid and apolipoprotein compositions, we noted compositional changes. In A-ILp, the levels of lipid, except for triglyceride, and the level of apoC-III were significantly higher in IDDM. In LpA-I/A-II, the levels of lipids, except for triglyceride, the levels of apoC-III, and the ratio of apoA-I to apoA-II were significantly higher in IDDM. In lipoprotein containing apoA-I but no apoA-II, the levels of all lipids and apolipoproteins in IDDM were similar to those in the controls. The percent phospholipid in A-ILp and LpA-I/A-II was significantly higher in IDDM. All of these changes of A-ILp are similar to those associated with the reduced risk in the nondiabetic population. However, apolipoprotein changes of LpA-I/A-II may possibly be related to the accelerated atherosclerotic processes noted in patients with IDDM. (Pediatr Res 28: 42 45, 1990)
\end{abstract}

\section{Abbreviations}
A-ILp, lipoprotein containing apoA-I
LpA-I/A-II, lipoprotein containing apoA-I and apoA-II
LpA-I, lipoprotein containing apoA-I but no apoA-II
HDL-C, HDL-cholesterol
TC, total cholesterol
TG, triglyceride
PL, phospholipid
$\mathrm{CE}$, cholesteryl ester
IDDM, insulin-dependent diabetes mellitus
NIDDM, noninsulin-dependent diabetes mellitus

The atherosclerotic process is accelerated in patients with IDDM, and atherosclerotic cardiovascular disease is one of the major causes of death in these patients $(1,2)$. Several research groups have shown that plasma levels of HDL inversely correlate with the incidence of atherosclerotic cardiovascular disease in cases of NIDDM, similar to findings in the nondiabetic populations (3-5). However, to the best of our knowledge, there are no

Received November 6. 1989; accepted February 26, 1990.

Reprint requests: T. Ohta, M.D., Ph.D., Department of Pediatrics, Kumamoto University Medical School, ]-[-], Honjo, Kumamoto City, Kumamoto 860, Japan. such data related to IDDM. Differing from NIDDM with lower HDL-C levels, plasma HDL-C levels in IDDM are either normal or elevated $(6,7)$. These lines of evidence suggest that factors other than HDL are responsible for the accelerated atherosclerotic process seen in IDDM. Should compositional changes of HDL be present in IDDM, then HDL would lose physiologic functions such as reverse cholesterol transport. There are data on the HDL composition of IDDM (8-10); however, most workers use $\mathrm{HDL}$ isolated by ultracentrifugation, a method that inevitably leads to a significant loss of apolipoproteins and lipids (11). We attempted to isolate a more native HDL than that obtained by conventional ultracentrifugation.

We developed antiapoA-I and antiapoA-II immunoaffinity columns using a formylated derivative of cellulose gel (12-14) and we isolated and characterized two species of A-ILp from 12 girls with IDDM: LpA-I/A-II, and LpA-I. These lipoproteins are assumed to be the more native form of HDL than is the HDL isolated by ultracentrifugation (12-15). The results were compared with findings in age- and sex-matched controls.

\section{MATERIALS AND METHODS}

Subjects. The clinical data on the 12 Japanese girls with typical IDDM are summarized in Table 1 . The ages ranged from 6-15 $y$. The diagnosis of IDDM was based on the criteria of National Diabetes Data Group (16). All had been diagnosed 0.5-5 y earlier and were clinically stable at the time of the study. Insulin (a combination of intermediate and rapid human insulin) was given s.c. twice a day. All patients were on adequate diets (caloric intake: $50 \%$ from carbohydrate, $30 \%$ from fat, $20 \%$ from protein). Nineteen nondiabetic healthy female subjects from the same part of Japan (Kumamoto-City) were the controls. They were volunteers from children of the staff at Kumamoto University. Age distribution in the control group was similar to that of the patients. Their lipid and apolipoprotein levels (Table 2) were all within mean $\pm \mathrm{SD}$ of those of healthy female children at Kumamoto-City. None of the study subjects had renal failure or bepatic or thyroid abnormalities and no child was taking any drug (except for insulin in the IDDM subjects). Blood samples were obtained from all of these subjects after an overnight fast, then the insulin was injected and breakfast provided. Informed consent was obtained from each subject and from parents, as required. Venous blood $(10 \mathrm{~mL})$ was drawn into vacutainer tubes containing disodium EDTA $(1.5 \mathrm{mg} / \mathrm{mL})$. Upon separation of the plasma at $4^{\circ} \mathrm{C}, 1500 \times g$ for $15 \mathrm{~min}$, sodium azide and gentamicin were added as preservatives in final concentration of $2.0 \mu \mathrm{g} / \mathrm{mL}$ and $5.0 \mu \mathrm{g} / \mathrm{mL}$, respectively.

Methods. Isolation of two species of A-ILp using immunosorbent column. Two species of A-ILp were isolated from plasma 
Table I. Clinical data on diabetic patients and controls*

\begin{tabular}{lcccccc}
\hline & Age $(\mathrm{y})$ & Sex & IBW $(\%)$ & $\begin{array}{c}\text { Insulin dose } \\
(\mathrm{IU} / \mathrm{kg})\end{array}$ & $\begin{array}{c}\text { Fasting glucose } \\
(\mathrm{mg} / \mathrm{dL}) \dagger\end{array}$ & $\begin{array}{c}\text { HbA } \\
(\%)\end{array}$ \\
\hline Diabetics & $12.3 \pm 2.6$ & $\mathrm{~F}$ & $107.0 \pm 5.5$ & $1.0 \pm 0.2$ & $140 \pm 32$ & $8.2 \pm 0.5$ \\
Controls & $12.6 \pm 3.0$ & $\mathrm{~F}$ & $109.3 \pm 5.3$ & 0 & $85 \pm 9$ & $4.7 \pm 0.2$ \\
\hline
\end{tabular}

* Values are means \pm SD. IBW, ideal body wt.

$\dagger$ To convert to mmol/L, multiply by 0.0556 .

Table 2. Plasma lipid and apolipoprotein levels*

\begin{tabular}{lcc}
\hline & Control & Diabetics \\
\hline TCt & $167.2 \pm 3.8$ & $187.5 \pm 7.8$ \\
CE $\$$ & $119.4 \pm 3.0$ & $134.7 \pm 5.9$ \\
TG $\$$ & $77.7 \pm 8.0$ & $95.7 \pm 11.2$ \\
PL\| & $189.9 \pm 3.1$ & $212.9 \pm 7.0$ \\
HDL-C $\ddagger$ & $57.7 \pm 2.9$ & $68.3 \pm 2.4$ \\
ApoA-I & $140.4 \pm 3.9$ & $145.8 \pm 4.7$ \\
ApoA-II & $32.6 \pm 0.9$ & $31.7 \pm 1.9$ \\
ApoC-II & $2.99 \pm 0.29$ & $3.62 \pm 0.48$ \\
ApoC-III & $7.66 \pm 0.36$ & $7.88 \pm 0.58$ \\
ApoE & $3.58 \pm 0.17$ & $3.96 \pm 0.25$ \\
\hline
\end{tabular}

* Values are mean \pm SEM $(\mathrm{mg} / \mathrm{dL})$.

$\dagger$ To convert to $\mathrm{mmol} / \mathrm{L}$, multiply by 0.0259 .

$\ddagger p<0.01$, significance between control and diabetics.

$\S$ To convert to $\mathrm{mmol} / \mathrm{L}$, multiply by 0.0113 .

$\| p<0.005$, significance between control and diabetics.

by a combination of antiapoA-I and antiapoA-II immunosorbent columns, as described $(12,13)$. Briefly, fresh plasma $(3 \mathrm{~mL})$ was applied on an antiapoA-I immunosorbent column. After washing extensively with $0.01 \mathrm{M}$ Tris, $0.5 \mathrm{M} \mathrm{NaCl}, 1 \mathrm{mM}$ EDTA, $\mathrm{pH}$ 7.5 (buffer A), the column was eluted with $0.1 \mathrm{M}$ acetic acid, I mM EDTA ( $\mathrm{pH} 3.0$ ). Each effluent was immediately adjusted to pH 7.4 with $1.0 \mathrm{M}$ Tris solution and dialyzed against $0.15 \mathrm{M}$ $\mathrm{NaCl}$ and 1 mM EDTA, pH 7.4 (buffer B). Finally, the sample was concentrated to $9 \mathrm{~mL}$ in an ultra-filtration cell (Amicon Corp., Danvers, ME) equipped with PM-10 membrane. A portion ( $3 \mathrm{~mL}$ ) of this sample was used for analysis of apolipoproteins and lipids. The remaining portion $(6 \mathrm{~mL})$ was used to separate the A-ILp into LpA-I/A-II and LpA-I. The sample was applied on an antiapoA-II immunosorbent column. The column was washed with buffer $A$ to obtain the unbound fraction (LpAI). The bound fraction (LpA-I/A-II) was eluted with $0.1 \mathrm{M}$ acetic acid, $1 \mathrm{mM}$ EDTA, $\mathrm{pH}$ 3.0. Both the unbound and bound fractions were dialyzed and then concentrated with an ultrafiltration apparatus (Amicon) to $6 \mathrm{~mL}$ in buffer $\mathrm{B}$, respectively.

Protein and lipid analysis. The apoA-I, apoA-II, apoC-II, apoC-III, and apoE concentrations of plasma, A-ILp, LpA-I/AII, and LpA-I were measured by radial immunodiffusion assay, as described (17). Cholesterol and TG concentrations of these samples were analyzed on an ABA 100 Autoanalyzer (Abbott Laboratories, N. Chicago, IL), using enzymatic methods $(18,19)$. CE was measured by enzymatic methods and fluorometry (20). HDL-C was determined by the method of Lopes-Virella $e t$ al. (21) after precipitation with sodium phosphotungstate and magnesium chloride. Phospholipid was analyzed by the method of Bartlett (22). The protein content of each fraction from the immunosorbent columns was determined by the method of Lowry et al. (23).

Electrophoretic analysis. Agarose gel electrophoresis was performed using a Pol-E Film system for lipoprotein electrophoresis (Corning Medical, Medfield, MA) at pH 8.6, followed by staining with Fat Red 7B. Slab gel electrophoresis of lipoproteins isolated in $15 \%$ polyacrylamide gels containing $0.1 \%$ SDS was performed according to Weber and Osborn (24). The Stokes diameters of the particles isolated were estimated by gradient PAGE on precast polyacrylamide $4 / 30$ gels (Pharmacia, Inc., Piscataway, NJ) according to the procedure provided by the manufacturer. Thyroglobulin, apoferritin, catalase, lactate dehydrogenase, and bovine albumin (Pharmacia) were used as the calibrating proteins. The Stokes diameters of these calibrating proteins are: thyroglobulin, $17.0 \mathrm{~nm}$; apoferritin, $12.2 \mathrm{~nm}$; catalase, $10.4 \mathrm{~nm}$; lactate dehydrogenase, $8.2 \mathrm{~nm}$; and bovine albumin, $7.5 \mathrm{~nm}$.

Statistical Evaluation. The Wilcoxon signed rank test was used to evaluate the data.

\section{RESULTS}

In a previous report (14), we found no compositional differences in lipoproteins containing apoA-I between prepubertal and pubertal girls. Thus, we treated our subjects studied as one group.

Plasma lipid and apolipoprotein levels. Plasma lipid and apolipoprotein levels are summarized in Table 2. The levels of TC, CE, PL, and HDL-C in IDDM were significantly higher than those in control subjects $(p<0.01$ or 0.005$)$. The TG and all apolipoprotein levels were similar in IDDM and control subjects.

Lipid and apolipoprotein compositions of $A-I L p$. The lipid and apolipoprotein compositions of A-ILp are shown in Table 3. TC, CE, PL, and apoC-III levels in IDDM were significantly higher than those in control subjects $(p<0.05$ or 0.01$)$. Other components were similar in IDDM and control subjects. The molar ratio of apoA-I to apoA-II was slightly higher in IDDM, albeit with no statistical significance.

Lipid and apolipoprotein compositions of LpA-I/A-II. The lipid and apolipoprotein components of LpA-I/A-II are summarized in Table 4. The levels of TC, CE, PL, and apoC-III were significantly higher in IDDM than in control subjects $(p<0.05,0.01$, or 0.005). TG and other apolipoprotein levels were similar in IDDM and control subjects. The molar ratio of apoA-I to apoAII was significantly higher in IDDM than in control subjects ( $p$ $<0.05$ ).

Lipid and apolipoprotein compositions of $L p A-I$. The lipid and apolipoprotein compositions of LPA-I are summarized in Table 5. The levels of all lipids and apolipoproteins were similar in IDDM and control subjects.

Percent lipid compositions of $A-I L p, L p A-I / A-I I$ and $L p A-I$. As shown in Table 6, percentages of PL in A-ILp and LpA-I/AII were significantly higher in IDDM than those in control

Table 3. Lipid and apolipoprotein concentrations of $A-I L p^{*}$

\begin{tabular}{lcc}
\hline & Control & Diabetics \\
\hline TC† $\ddagger$ & $71.3 \pm 3.9$ & $80.3 \pm 3.6$ \\
CE $\ddagger$ & $52.4 \pm 2.2$ & $59.8 \pm 2.6$ \\
TG\$ & $18.3 \pm 1.6$ & $17.3 \pm 1.8$ \\
PL\| & $115.0 \pm 4.0$ & $132.5 \pm 4.7$ \\
ApoA-I & $140.3 \pm 3.8$ & $145.2 \pm 4.6$ \\
ApoA-II & $32.7 \pm 0.9$ & $31.7 \pm 1.8$ \\
ApoA-I/ApoA-II & $2.66 \pm 0.06$ & $2.91 \pm 0.16$ \\
ApoC-II & $1.49 \pm 0.14$ & $1.73 \pm 0.17$ \\
ApoC-III & $4.28 \pm 0.26$ & $5.34 \pm 0.44$ \\
ApoE & $1.49 \pm 0.15$ & $1.54 \pm 0.18$ \\
\hline
\end{tabular}

* Values are mean \pm SEM (mg/dL), except for apoA-l/apoA-ll, which is a molar ratio (molecular wt of apoA-1 and apoA-II are 28300 and 17400 , respectively).

$\dagger$ To convert to $\mathrm{mmol} / \mathrm{L}$, multiply by 0.0259 .

$\ddagger p<0.05$, significance between control and diabetics.

$\S$ To convert to $\mathrm{mmol} / \mathrm{L}$, multiply by 0.0113 .

$\| p<0.01$, significance between control and diabetics. 